J. Clin. Chem. Clin. Biochem.

Vol. 20, 1982, pp. 313-318

\title{
Vergleich kommerziell erhältlicher Radioimmunoassays zur Bestimmung von Gallensäuren im Serum
}

\author{
Von H. J. Wildgrube, W. Schiller, M. Winkler, J. Weber, H. Campana und G. Mauritz \\ Technische Mitarbeit: $H$. Stockhausen

\begin{abstract}
Abteilung für Gastroenterologie (Leiter: Prof. Dr. M. Classen) Zentrum der Inneren Medizin der Johann-Wolfgang-Goethe-Universität Frankfurt/Main
\end{abstract}

(Eingegangen am 7. September/7. Dezember 1981)

Zusammenfassung: Drei kommerziell erhältliche Radioimmunoassays zur Bestimmung von Gallensäuren im Serum werden hinsichtlich ihrer Spezifität und Präzision bewertet. Der SLCG-Radioimmunoassay (Fa. Abbott) erfaßt das am engsten definierte Gallensäurespektrum (sulfatierte Glykolithocholsäure), der CG-Radioimmunoassay (Fa. Abbott) vornehmlich Cholsäurekonjugate und der CBA-Radioimmunoassay (Fa. Beçton-Dickinson) alle konjugierten Gallensäuren, wobei Taurinmetaboliten überrepräsentiert sind. In Bezug auf die Kreuzreaktionen bestehen bedeutsame Unterschiede zu den Angaben des Herstellers für den CG-Radioimmunoassay, der zu 32\% Taurocholsäure erfaßt, sowie für den CBA-Radioimmunoassay, der Taurochenodesoxycholsäure nur zu $118 \%$ ermittelt. Die Wiederfindungsrate definierter Cholansäuren liegt bei physiologischen Albumin-Globulin-Konzentrationen zwischen 85 und $101 \%$. Gute Reproduzierbarkeit wird für den CG-Radioimmunoassay im Bereich von 0,5 bis 10,0 $\mu \mathrm{mol} / 1$, für den CBARadioimmunoassay im Bereich von 1,0 bis $25,0 \mu \mathrm{mol} / 1$ und für den SLCG-Radioimmunoassay im Bereich von 0,5 bis $3,0 \mu \mathrm{mol} / 1$ gefunden. Für die Inter- und Intraassay-Präzision bestehen zwischen den drei Radioimmunoassays keine nenneswerten Unterschiede.

\section{Comparison of commercially available radioimmunoassays for the determination of bile acids in serum}

Summary: Three commercially available radioimmunoassays for the determination of bile acids in serum were evaluated with respect to specificity and precision. The SLCG-radioimmunoassay (Abbott) measures only sulphated glycolithocholic acid, the CG-radioimmunoassay (Abbott) measures chiefly cholic acid conjugates, and the CBA-radioimmunoassay (Becton-Dickinson) measures all conjugated bile acids, with an over-response to taurine metabolites. With respect to cross reactions, the performances of the CG-and the CBA-radioimmunoassays differed significantly from those stated by the manufacturers, the former showing a $32 \%$ response to taurocholic acid, the latter responding only $118 \%$ to taurochenodeoxycholic acid. At physiological concentrations of albumin + globulin, the recovery of defined cholanic acids was $85-101 \%$. Good reproducibility was shown by the CG-radioimmunoassay in the range $0.5-10.0 \mu \mathrm{mol} / 1$, by the CBA-radioimmunoassay in the range $1.0-25.0 \mu \mathrm{mol} / 1$, and by the SLCG-radioimmunoassay in the range $0.5-3.0 \mu \mathrm{mol} / 1$. There were no important differences in the inter- and intra-assay precision of the three methods.

\section{Einfưhrung}

Die Bestimmung der im Serum zirkulierenden Gallensäuren hat zunehmendes Interesse gefunden, nachdem radioimmunologische Verfahren praktikable Analysen ermöglichen. Seit 1973, mit der ersten Beschreibung eines Gallensäuren-Radioimmunoassays durch Simmonds et al. (1), sind verschiedene Modifikationen erprobt und inzwischen auch kommerziell angeboten worden. Die vorliegende Untersuchung hat zum Ziel, drei dieser standardisierten Radioimmunoassays zu charakterisieren und miteinander zu vergleichen.

\section{Material und Methodik}

Die von uns geprüften Radioimmunoassays sind

1. der CG-Radioimmunoassay (Radioimmunoassay for the determination of cholylglycine in serum), Fa. Abbott Laboratories, Diagnostic Division, North Chicago; 
2. der Conjugated bile acids solid phase radioimmunoassay kit (CBA-Radioimmunoassay), Fa. Becton-Dickinson and Co., Orangeburg, New York;

3. der SLCG-Radioimmunoassay (Sulfolithocholylglycine radioimmunoassay), Fa. Abbott Laboratories, Diagnostics Division, North Chicago.

Das Antigen dieser Radioimmunoassays ist mit ${ }^{125}$ Iod markiert. Gemessen wird stets die gebundene Fraktion. Der CBA-Radioimmunoassay zeichnet sich durch Verwendung Antikörperbeschichteter Röhrchen aus (Tab. 1 und 2).

Die Untersuchungen zur Richtigkeit und Präzision sind mit Poolseren bekannter Gallensäurenkonżentrationen, gallensäurenfreiem Poolserum und Albumin-Globulin-Pufferlösungen vorgenommen worden. Die Poolseren enthalten Gesamtgallensäuren von $5,3,14,8$ und $26,4 \mu \mathrm{mol} / 1$, enzymatisch-fluorimetrisch gemessen (Sterognost-3 RRA, Fa. Nyegaard und Co., Oslo). Gallensäurenfreies Serum ist aus Poolserum durch Zugabe von Aktivkohle hergestellt worden (1). Die Pufferlösurigen bestehen aus $50 \mathrm{mmol}$ Phosphat und $9 \mathrm{~g} \mathrm{NaCl}$ je Liter unter Zugabe von Human-Albumin (200 g/l, Blutspende Frankfurt/Main) und $\gamma$-Globulin aus Rinderserum (Fa. Serva, Heidelberg). Dabei sind Albuminlösungen $(20,30$ und $70 \mathrm{~g} / \mathrm{l})$ sowie Lösungen mit 20 , 30 und $70 \mathrm{~g} / 1$ Albumin, die auch 10,20 und $30 \mathrm{~g} / 1$ Globulin enthielten, geprüft worden.

Zur Bestimmung der Kreuzreaktionen sind folgende mittels: Dünnschichtchromatographie oder HPLC überprüfte Testsubstanzen verwendet worden:

Glykocholsäure, Na-Salz,

Glykochenodesoxycholsäure Na-Salz,

Glykodesoxycholsäure Na-Salz,

Taurocholsäure $\mathrm{Na}-\mathrm{Salz}$,

Taurodesoxycholsäure Na-Salz,

Chenodesoxycholsäure,

Desoxycholsäure,

Glykodehydrocholsäure Na-Salz,

Ursodesoxycholsäure,

Taurohyodesoxycholsäure,

17-Ketosteroide Mix (Fa. Supelco Inc., Bellefonte, Pennsylvania),

Glykolithocholsäure Na-Salz,

Glykoursodesoxycholsäure Na-Salz,

Tauroursodesoxycholsäure Na-Salz (Calbiochem, La Jolla, California),

Taurochenodesoxycholsäure Na-Salz,

Taurolithocholsäure,

Lithocholsäure,

Sulfoglykolithocholsäure,

Sulfolithocholsäure,

Sulfochenodesoxycholsäure,

Sulfodesoxycholsäure (PL. Biochemicals Inc., Milwaukee),

Hyodesoxycholsäure,

3,12-Diketocholansäure

7,12-Diketolithocholsäure,

Coprostanol (Appl. Science Europe, Oud-Beijerland/Holland),

Cholsäure,

Cholesterin (Fa. Serva, Heidelberg),

Dehydrocholsäure,

$3 \alpha$-Hydroxy-7,12-diketocholansäure,

3,7-Dihydroxy-12-ketocholansäure (Fa. Casella, Frankfurt/Main).

Als Kontrollen finden Poolserum und Pufferlösungen nach Ein waage definierter Mengen an Glykocholsäure und sulfatierter Glykolithocholsäure Verwendung. Der Proteinanteil der Pufferlösungen besteht aus Albumin und $\gamma$-Globulin aus Rinderserum (Fa. Serva, Heidelberg).

Die Berechnung der Standardkurve erfolgt durch Log-LogitTransformationen. Die minimal meßbaren Konzentrationen sind mittels der von Rodbard \& Lewald (3) angegebenen Methode ermittelt worden.

\section{Ergebnisse}

\section{Standardkurve}

Die anhand von jeweils 28 Untersuchungen gewonnenen Standardkurven zeigen, daß die angestrebte Linearitäät nicht im gesamten, vom Hersteller angegebenen Konżentrationsbereich gewährleistet isst. Gleichwohl liegen die beim Gesunden zu erwartenden Werte im günstigen Meßbereich (Abbilđung 1). Die Inkubationszeiten sollen für den CG-Radioimmunoasșay und den CBA-Radioimmunoassay bei $37^{\circ} \mathrm{C}$ mindestens eine Stunde betragen, der
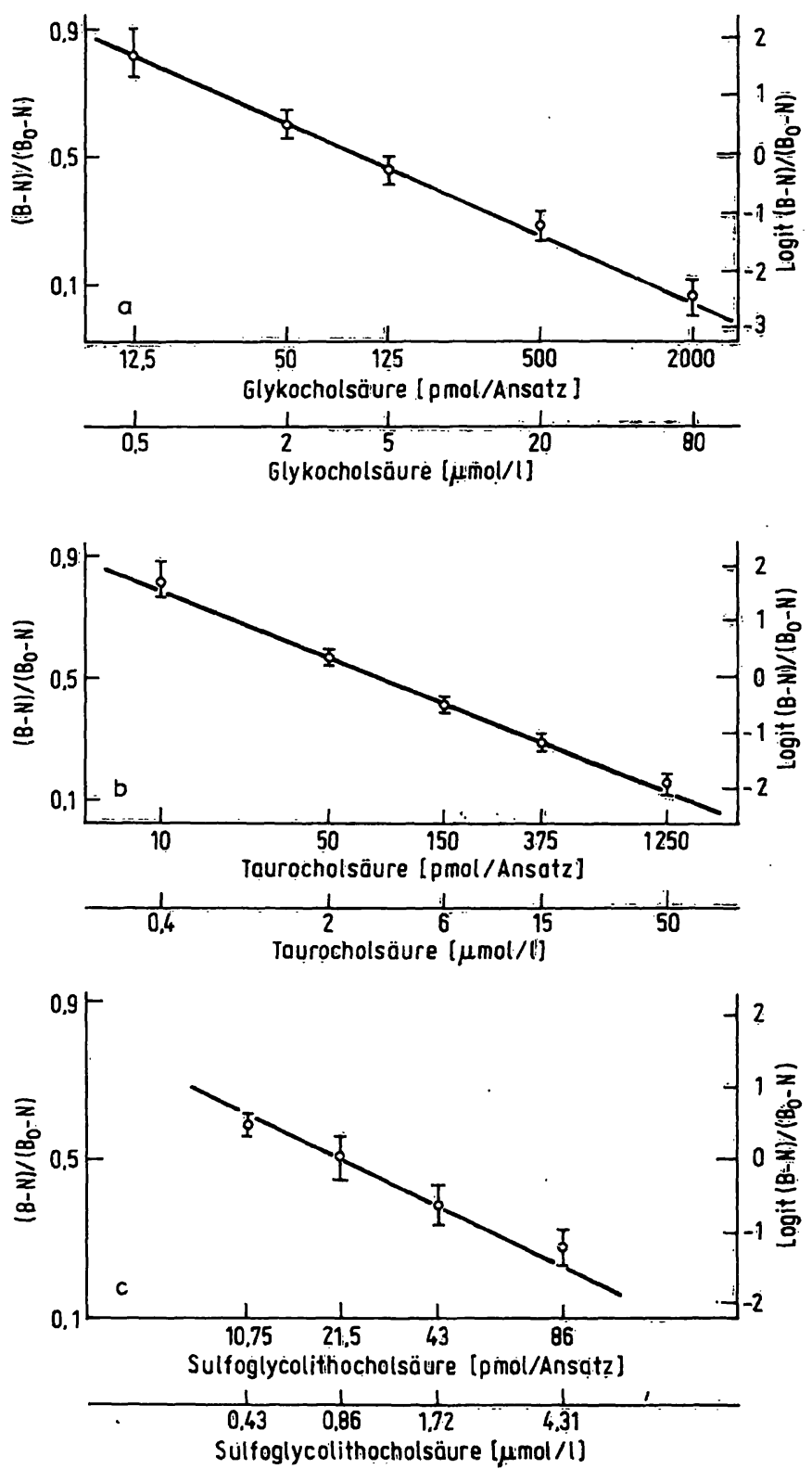

Abb. 1. Standardkurven

(Mittelwerte und Standardabweichungen aus jeweils 28 Tests).

a) für Glykocholsäure im CG-Radioimmunoassay;

b) für Taựocholsäure im CBA-Radioimmunoassay;

c) für Sulfoglykolithocholsäure im SLCG-Rádioimmunoassay.

- Standardlösungen gemäß Angaben der Herstellèr,

(B: Antikörpergebundene Fraktion,.

N: Unspezifịsch gebundene Fraktioh). 
Tab. 1. Allgemeine Angaben zu den drei geprüften Radioimmunoassays für Gallensäuren im Serum.

\begin{tabular}{llll}
\hline & CG-Radioimmunoassay & CBA-Radioimmunoassay & SLCG-Radioimmunoassay \\
\hline Packungsgröße & $100 / 300$ & 100 & $100 / 300$ \\
Radioaktivität kBq je Packung & $380 / 1140$ & 185 & $380 / 1140$ \\
Bereich der Standardkurve & $0-4000 \mu \mathrm{g} / 100 \mathrm{ml}$ & $0-50 \mu \mathrm{mol} / \mathrm{l}$ & $0-250 \mu \mathrm{g} / 100 \mathrm{ml}$ \\
& $=0-80 \mu \mathrm{mol} / \mathrm{l}$ & & $0-4,31 \mu \mathrm{mol} / 1$ \\
Zahl der Standards & 6 & 6 & 6 \\
Art des Standards & Glykocholsäure & Taurocholsäure & Sulfoglykolithocholsäure \\
Verdünnungslösung & 0-Standard & $0-$-Standard & $0-$ Standard \\
Kontrollserum & nein & nein & nein \\
\hline
\end{tabular}

Tab. 2. Arbeitsschema der drei geprüften Radioimmunoassays.

\begin{tabular}{lccc}
\hline & CG-Radioimmunoassay & CBA-Radioimmunoassay & SLCG-Radioimmunoassay \\
\hline Serumprobe & $25 \mu \mathrm{l}$ & $25 \mu \mathrm{l}$ & $25 \mu \mathrm{l}$ \\
Antigen & $200 \mu \mathrm{l}$ & $1000 \mu \mathrm{l}$ & $200 \mu \mathrm{l}$ \\
Antikörper & $200 \mu \mathrm{l}$ & nicht erforderlich & $200 \mu \mathrm{l}$ \\
Inkubationstemperatur & $37^{\circ} \mathrm{C}$ & $37^{\circ} \mathrm{C}$ & $25^{\circ} \mathrm{C}$ \\
Inkubationszeit & $60 \mathrm{Minuten}$ & 60 Minuten & $60 \mathrm{Minuten}$ \\
Trennung von gebundenem & a) $2000 \mu \mathrm{l}$ Polyethylenglykol & a) Überstand dekantieren & a) $2000 \mu 1$ Polyethylenglykol \\
und freiem Antigen & $(180 \mathrm{ml} / \mathrm{l})$ & b) $1000 \mu \mathrm{H} \mathrm{H}_{2} \mathrm{O}$ und erneut & b) 10 min mit $1000 \mathrm{~g}$ \\
& b) 10 min mit $1000 \mathrm{~g}$ & dekantieren & zentrifugieren \\
Meßzeit/Röhrchen & zentrifugieren & & c) Überstand dekantieren \\
\hline
\end{tabular}

SLCG-Radioimmunoassay kann bei Raumtemperatur angesetzt werden. Die Verlängerung der Inkubationszeit bis zu 8 Stunden hat keinen Einfluß auf die Meßergebnisse. Nach Inkubation bei $+4{ }^{\circ} \mathrm{C}$ erreichen CG- und SLCG-Radioimmunoassay ihr Reaktionsgleichgewicht nach 20 Stunden, während der CBA-Radioimmunoassay zu dieser Zeit noch nicht stabilisiert ist.

Änderungen der Proteinkonzentrationen im Serum, wie sie beim kranken Menschen zu beobachten sind, nämlich niedrige Albuminkonzentrationen und hohe $\boldsymbol{\gamma}$-Globulinanteile, haben keinen Einfluß auf die Reaktionskinetik. In 20,30 und $70 \mathrm{~g} / 1$ Albuminlösung mit Z̈usatz von jeweils 10 bis $30 \mathrm{~g} / 1 \gamma$-Globulin werden die Standards bei allen Radioimmunoassays in der eingewogenen Menge erfaßt. Im Gegensatz hierzu haben die Unter= suchungen mit reinen Albuminlösungen erkennen lassen, daß bei Konzentrationen unter $35 \mathrm{~g} / 1$ falsch niedrige Gallensäurewerte bestimmint werden.

\section{Richtigkeit und Präzision}

Zur Ermittlung der Wiederfindungsrate sind Glykocholsäure und sulfatierte Glykolithocholsäure in fün Konzentrationen zwischen 0,1 und $400 \mu \mathrm{mol} / 1$ Pufferlösung mit $40 \mathrm{~g} / 1$ Albumin und $10 \mathrm{~g} / 1 \mathrm{\gamma}$-Globulin den drei Poolseren mit bekanntem Gallensäurengehalt und gallensäurenfreiem Serum zugesetzt worden. Glykocholsäure wird zu durchschnittlich 97,8\% im CG-Radioimmunoassay und $\mathrm{zu} 85 \%$ im CBA-Radioimmunoassay wiedergefunden. Für sulfatierte Glykolithocholsäure beträgt die Wiederfindung im SLCG-Radioimmunoassay $101 \%$.

Für die Inter- sowie Intraassay-Präzision (Tab. 3) werden nur graduelle Unterschiede zwischen den geprüften Radioimmunoassays erkennbar. Die minimal meßbaren Konzenträtiönen können mit $0,22 \pm 0,07 \mu \mathrm{mol} / 1$ für den CGRadioimmunoassay, 0,19 $\pm 0,05 \mu \mathrm{mol} / 1$ für den CBARadioimmunoassay und $0,11 \pm 0,04 \mu \mathrm{mol} / 1$ für den SLCG-Radioimmunoassay ermittelt werden. Die unspezifische Bindung beträgt in allen drei Tests weniger als $0,05(\mathrm{~B} / \mathrm{T})$. Die Konzentration im Bereich des 50\% Interzepts $\left(B / B_{0}=0,5\right) \mathrm{kann}$ mit 3,7 $\pm 0,29 \mu \mathrm{mol} / 1 \mathrm{im}$ CG-Radioimmunoassay, $3,0 \pm 0,52 \mu \mathrm{mol} / 1 \mathrm{im} \mathrm{CBA}$ Radioimmunoassay und $0,86 \pm 0,07 \mu \mathrm{mol} / 1 \mathrm{im} \mathrm{SLCG-}$ Radioimmunoassay berechnet werden.

\section{Kreuzreaktionen}

Die Kreuzreaktionen sind in Pufferlösungen, die $40 \mathrm{~g} / 1$ Humanalbumin und $10 \mathrm{~g} / 1$ Rinder- $\gamma$-Globulin enthielten, in Konzentrationen von 0,1 bis $400 \mu \mathrm{mol} / 1$ gemessen und gemäß den Angaben von $A b r a h a m$ et al. (4) berechnet worden (Tab. 4). Die Analysen beruhen auf den Mittelwerten von jeweils 6 Ansätzen für jeden Radioimmunoassay. Zusätzlich zu den in dieser Tabelle aufgeführten Gallensäuren sind in den gleichen Konzentrationen chemisch verwandte Metaboliten geprüft worden, nämlich Glykodehydrocholsäure, Dehydrocholsäure, 
Tab. 3. Präzision der Radioimmunoassays gemessen mit verschiedenen Poolseren

( $\bar{x}$ : Mittelivert, s: Standardabweichung, VK: Variationskoeffizienf, n: Anzahl der Untersuchungen).

\begin{tabular}{|c|c|c|c|c|c|c|c|c|c|}
\hline & $\begin{array}{l}\bar{x} \\
(\mu \mathrm{mol} / \mathrm{l})\end{array}$ & $\begin{array}{l}\mathrm{s} \\
(\mu \mathrm{mol} / \mathrm{l})\end{array}$ & $\begin{array}{l}\text { VK } \\
(\%)\end{array}$ & $\mathbf{n}$ & $\begin{array}{l}\bar{x} \\
(\mu \mathrm{mol} / \mathrm{l})\end{array}$ & $\begin{array}{l}s \\
(\mu \mathrm{mol} / \mathrm{l})\end{array}$ & $\begin{array}{l}\text { VK } \\
(\%)\end{array}$ & & $\mathrm{n}$ \\
\hline CG-Ria & $\begin{array}{l}1,36 \\
2,89\end{array}$ & $\begin{array}{l}0,16 \\
0,26\end{array}$ & $\begin{array}{r}12 \\
9\end{array}$ & $\begin{array}{l}28 \\
26\end{array}$ & $\begin{array}{l}1,25 \\
2,79\end{array}$ & $\begin{array}{l}0,05 \\
0,14\end{array}$ & $\begin{array}{l}4 \\
5\end{array}$ & $: 1$ & $\begin{array}{l}42 \\
32\end{array}$ \\
\hline CBA-Ria & $\begin{array}{r}3,21 \\
8,93 \\
14,71\end{array}$ & $\begin{array}{l}0,51 \\
1,19 \\
2,20\end{array}$ & $\begin{array}{l}16 \\
13 \\
15\end{array}$ & $\begin{array}{l}24 \\
19 \\
15\end{array}$ & $\begin{array}{r}3,07 \\
8,57 \\
15,62\end{array}$ & $\begin{array}{l}0,27 \\
0,44 \\
1,09\end{array}$ & $\begin{array}{l}9 \\
5 \\
7\end{array}$ & & $\begin{array}{l}28 \\
18 \\
22\end{array}$ \\
\hline SLCG-Ria & $\begin{array}{l}1,20 \\
3,04\end{array}$ & $\begin{array}{l}0,15 \\
0,21\end{array}$ & $\begin{array}{r}12 \\
7\end{array}$ & $\begin{array}{l}30 \\
24\end{array}$ & $\begin{array}{l}1,17 \\
3,19\end{array}$ & $\begin{array}{l}0,04 \\
0,22\end{array}$ & $\begin{array}{l}3 \\
7\end{array}$ & & $\begin{array}{l}40 \\
28\end{array}$ \\
\hline
\end{tabular}

Tab. 4. Kreuzreaktionen der geprüften Radioimmunoassays

(A: Angaben des Herstellers, B: eigene Werte, berechnet aus den Mittelwerten von jeweils 6 Ansätzen in Konzentrationen von $0,1,1,0,10,0,100,0$ und $400,0 \mu \mathrm{mol} / 1$, n.a.: nicht angegeben).

\begin{tabular}{|c|c|c|c|c|c|c|}
\hline \multirow[t]{2}{*}{ Substanz } & \multicolumn{2}{|c|}{ CG-Ria } & \multicolumn{2}{|c|}{ CBA-Ria } & \multicolumn{2}{|c|}{ SLCG-Ria } \\
\hline & A & B & A & B & $\mathbf{A}$ & B \\
\hline $\begin{array}{l}\text { Glykocholsäure } \\
\text { Glykochenodesoxycholsäure } \\
\text { Glykodesoxycholsäure } \\
\text { Glykolithocholsäure } \\
\text { Glykoursodesoxycholsäure }\end{array}$ & $\begin{array}{r}100 \\
14,5 \\
2,8 \\
0,4 \\
\text { n.a. }\end{array}$ & $\begin{array}{c}100 \\
12,9 \\
2,9 \\
1 \\
1\end{array}$ & $\begin{array}{l}85 \\
62 \\
3,3 \\
\text { n.a. } \\
\text { n.a. }\end{array}$ & $\begin{array}{c}78 \\
49 \\
3,8 \\
1 \\
2,3\end{array}$ & $\begin{array}{l}0 \\
0,05 \\
0,02 \\
0,40 \\
\text { n.a. }\end{array}$ & $\begin{array}{l}0 \\
i \\
1 \\
1 \\
0,12\end{array}$ \\
\hline $\begin{array}{l}\text { Taurocholsäure } \\
\text { Taurochenodesoxycholsäure } \\
\text { Taurodesoxycholsäure } \\
\text { Taurolithocholsäure } \\
\text { Tauroursodesoxycholsäure }\end{array}$ & $\begin{array}{r}16,5 \\
3,2 \\
1,7 \\
0,3 \\
\text { n.a. }\end{array}$ & $\begin{array}{c}31,9 \\
4,9 \\
1,1 \\
1 \\
1\end{array}$ & $\begin{array}{l}100 \\
210 \\
\quad 4,0 \\
\text { n.a. } \\
\text { n.a. }\end{array}$ & $\begin{array}{l}100 \\
118 \\
5,0 \\
1 \\
3,7\end{array}$ & $\begin{array}{l}0 \\
0,01 \\
0,01 \\
0,1 \\
\text { n.a. }\end{array}$ & $\begin{array}{l}0 \\
1 \\
1 \\
0,4 \\
0,2\end{array}$ \\
\hline $\begin{array}{l}\text { Cholsäure } \\
\text { Chenodesoxycholsäure } \\
\text { Desoxycholsäure } \\
\text { Lithocholsäure } \\
\text { Ursodesoxycholsäure }\end{array}$ & $\begin{array}{r}7,8 \\
3,3 \\
1,0 \\
0,1 \\
\text { n.a. }\end{array}$ & $\begin{array}{l}6,7 \\
1,3 \\
1 \\
1 \\
0\end{array}$ & $\begin{array}{r}3,1 \\
8,1 \\
0,2 \\
0,7 \\
\text { n.a. }\end{array}$ & $\begin{array}{l}1 \\
3,6 \\
1 \\
1 \\
0\end{array}$ & $\begin{array}{l}0 \\
0,05 \\
0 \\
0,05 \\
\text { n.a. }\end{array}$ & $\begin{array}{l}0 \\
1 \\
0 \\
0 \\
0\end{array}$ \\
\hline $\begin{array}{l}\text { Sulfoglykolithocholsäure } \\
\text { Sulfolithocholsäure } \\
\text { Sulfochenodesoxycholsäure } \\
\text { Sulfodesoxycholsäure }\end{array}$ & $\begin{array}{l}0 \\
0 \\
0 \\
0\end{array}$ & $\begin{array}{l}0 \\
0 \\
0 \\
0\end{array}$ & $\begin{array}{l}\text { n.a. } \\
\text { n.a. } \\
\text { n.a. } \\
\text { n.a. }\end{array}$ & $\begin{array}{l}0 \\
0 \\
1 \\
0\end{array}$ & $\begin{array}{r}71 \\
13,9 \\
4,3 \\
\text { n.a. }\end{array}$ & $\begin{array}{c}100 \\
28,4 \\
6,4 \\
1\end{array}$ \\
\hline
\end{tabular}

Hyodesoxycholsäure, Taurohyodesoxycholsäure, 3,12Diketocholansäure, Coprostanol, Cholesterin und 17. Ketosteroide. Keiner dieser Metaboliten konkurriert in quantitativ bedeutsamen Mengen um die spezifischen Antiseren der getesteten Radioimmunoassays.

Gleichwohl bestehen zwischen den unterschiedlich ausführlichen Angaben der Hersteller und den hier ermittelten Daten Unterschiede.

Der CG-Radioimmunoassay weist eine doppelt so hohe Kreuzreaktion für Taurocholsäure auf wie angegeben. Im CBA-Radioimmunoassay hingegen ist die Überrepräsentierung der Taurochenodesoxycholsäure weniger ausgeprägt. Die Abweichungen für den SLCG-Radioimmunoassay unterscheiden sich möglicherweise nur durch die unterschiedliche Berechnung des SLCGStandards. Im Verlauf einer mehr als zweijährigen Beobachtungsperiode ist keine Veränderung der Kreuzreaktionen nachweisbar gewesen.

\section{Serum oder Plasma?}

Bei insgesamt 16 Probanden ist Blut sowohl in Glasröhrchen, Plastikröhrchen und in mit EDTA=Kaliumbeschichtete Röhrchen sowie unter Zugabe von Heparin $(0,2 \mathrm{ml}$ Liquemin auf $10 \mathrm{ml})$ und Natriumcitratlösung ( $38 \mathrm{~g} / 1 ; 1 \mathrm{ml}$ auf $10 \mathrm{ml}$ ) entnommen worden. Die gewonnenen Meßwerte haben keine außerhalb der üblichen Schwankungsbreite liegenden Konzentrationsunterschiede erbracht.

\section{Diskussion}

Im Serum des Menschen werden vornehmlich 4 Cholansäuren gefunden, nämlich Cholsäure, Chenodesoxycholsäure, Desóxycholsäure und Lithocholsäure (5.). Cholsäure und Chenodesoxycholsäure stellen żusammen den größtẹn Anteil mit etwa $80 \%$ aller Gallensäuren. Diese Chọlansäuren, die sich durch die Zahl der Hydroxylgruppen am Sterangerüst unterscheiden, sind größtenteils säureamidartig an Glycin oder Taúrin gebunden. 
Normalerweise überwiegen die Glycinkonjugate. Beim gesunden Erwachsenen variiert der Glycin-TaurinQuotient zwischen 2:1 und 4:1 (6). Relativ neu ist die Erkenntnis, daß analog zu den Steroiden neben Konjugaten auch nennenswerte Mengen an sulfatveresterten Metaboliten entstehen (7). Hierbei handelt es sich um relativ hydrophile Molekuile, die renal ausgeschieden werden können.

Für die Analyse der Gallensäuren im Serum haben sich bisher gaschromatographische und enzymatische Meßtechniken durchgesetzt. Gaschromatographisch werden überwiegend die einzelnen Cholansäuren, enzymatisch die $C_{3}$-hydroxylierten Gallensäuren, also freie und konjugierte Cholansäuren bestimmt. Sulfatierte Metaboliten können nur nach aufwendigen Präparationen dargestellt werden (8). Aus diesen methodischen Gründen sollten Radioimmunoassays diesen Bestimmungsverfahren überlegen sein. Die enge strukturelle Verwandtschaft der verschiedenen Gallensäuren limitiert jedoch die Spezifität des beim Kaninchen erzeugten Antikörpers. Erhebliche Kreuzreaktionen zwischen Glycin- und Taurinkonjugaten derselben Cholansäure und selbst zwischen verschiedenen Gallensäuren sind deshalb nicht zu vermeiden. Dies wiederum bedeutet: die radioimmunologisch ermittelten Daten sind nicht ohne weiteres auf gaschromatographisch oder enzymatisch gefundene Werte übertragbar.

Von den hier geprüften kommerziell erhältlichen Radioimmunoassays ișt der SLCG-Radioimmunoassay relativ spezifisch, da er nähezu ausschließlich sulfatierte Metaboliten erfaßt. Die beiden übrigen Radioimmunoassays messen ein voneinander abweichendes Gallensäurenspektrum. Im Hinblick auf die Kreuzreaktion muß der vom Hersteller als Glykocholat-Radioimmunoassay ausgebotene Kit (Fa. Abbott) als ein Test charäkterisiert werden, der auf beide im Serum vorkommenden Cholsäurekonjugate anspricht. Für experimentelle Untersuchungen bleibt zu berïcksichtigen, daß beispielsweise beim Hund, der nur wenig oder keine Glykocholsäure synthetisiert, mit zunehmender enzymatisch oder gaschromatographisch ermittelter.Gesamt-Galllenșäurenkonzentration auch der ,CG-Radioimmunoassay“" șteigende Werte bestätigt. Der Antịkörper deś CBA. Radioimmunoassays reagiert hingegen auf alle Konjugate von Cholsạure und Chenodesoxycholsäure, wọbei die Tauriṇmețaboliten überrepräsentiert werden. Diese Unterschiede in der Spezifität der Antikörper fuhren zu Konzentrationsangaben, die nicht ohne weiteres miteinander gleichgesetzt oder gar zur Geșamt-Gallensäuremenge addiert werden köñnenen.

Im Hinblick auf die praktische Durchführung bleibt bemerkenswert, daß keine ethanolische Extraktion der Gallensäuren erforderlich ist. Damit sind die hier beschriebenen Radioimmunoassays den von der $\mathrm{Fa}$. Nordiclab ausgebotenen Tests überlegen (2). CG- und SLCG-Radioimmunoassay unterscheiden sich durch die Inkubationstemperatur. Der CBA-Radioimmunoassay ist weniger zeitaufwendig, da ein Pipettierschritt und die Sedimentierung des Präzipitats fortfallen. Für jeden Radioimmunoassay gilt aber die Forderung, den durch die Standardkurven angegebenen Konzentrationsbereiche nicht voll auszuschöpfen. Konzentrationen unter $0,5 \mu \mathrm{mol} / 1 \mathrm{im} \mathrm{CG-} \mathrm{und} \mathrm{SLCG-Radioimmunoassay}$ und unter $1,0 \mu \mathrm{mol} / 1 \mathrm{im}$ CBA-Radioimmunoassay sind weniger exakt reproduzierbar. Werte über $3 \mu \mathrm{mol} / 1$ für den SCLG-Radioimmunoassay, $10 \mu \mathrm{mol} / 1$ für den CGRadioimmunoassay und $25 \mu \mathrm{mol} / 1$ für den CBA-Radioimmunoassay sollten nach entsprechender Probenverdünnung wiederholt werden.

Soweit uns bekannt, wird bisher kein brauchbares Kontrollserum mit einer definierten Konzentration an Glykocholsäure oder sulfatierten Gallensäuren ausgeboten. Für Qualitätskontrollen muß der Untersucher deshalb ein Poolserum mitfụihren.

Neue diagnostische Methoden implizieren die Frage nach ihrer klinischen Relevanz. Mannigfache Studien über den Gallensäurenstoffwechsel haben inzwischen hinreichend bewiesen, daß die nur in der Leberzelle synthetisierten Metaboliten vielfältige Funktionen wahrnehmen und demzufolge auch Störungen unterliegen (Übersicht bei Nair \& Kritchevsky (9)). Diese zu erkennen ist Zweck von Gallensäureanalysen im Serum. Der bisher erforderliche methodische, d.h. apparative und personelle Aufwand bei der gaschromatographischen und enzymatischen Analyse hat systematische Untersuchungen an größeren Kollektiven unmöglich gemacht. Selbst die von wenigen Gruppen publizierten unterschiedlichen radioimmunologischen Vèrfahren sind nur an kleinen Patientengruppen erprobt worden. Insofern ist die klinische Relevanz der radioimmunologischen Gallensäurenanalysen im Vergleich zu den herkömmlichen diagnostischen Methoden noch keineswegs eindeutig charakterisierbar. Verschiedene Studien lassen vermuten, daß mit dem SLCG-Radioimmunoassay gemessene Erhöhungen sehr empfindlich fortgeschrittene chronische Lebererkrankungen oder eine Cholestase anzeigen $(10,11)$. Die Aussagen der CG-bzw. CBA-Radioimmunoassays entsprechen den bisher bekannten Diskussionen erhöhter Gallensäurenkonzentrationen im Serum. Dabei bleibt unentschieden, ob der spezifischere CG-Radioimmunoassay dem CBARadioimmunoassay, der vornehmlich Taurinkonjugate erfaßt, überlegen oder gleichwertig ist. Der Nachweis erhöhter Gallenšäurenkonzentrationen im Serum ist in jedem Fall Hinweis auf eine manifeste Lebererkrankung.

\section{Literatur}

1. Simmonds, W. J., Korman, M. G., Go, V. L. W. \& Hofman, A. F. (1973) Gastroenterology 65, 705-711.

2. Källberg, M. \& Tobiasson, P. (1980) J. Clin. Chem. Clin. Biochem. 18, $491-495$.

J. Clin. Chem. Clin. Bioohem. / Vol. 20, 1982 / No. 5 
3. Rodbard, S. D. \& Lewald, J. E. (1970) Acta Endocrinol. Suppl. 147, 79-103.

4. Abraham, G. E., Odell, W. D., Edwards, R. \& Purdy, J. M. (1970) Acta Endocrinol. Suppl. 147, 332-346.

5. Sandberg, D. H., Sjövall, J. \& Turner, D. A. (1965) J. Lipid Res. 6, 182-192.

6. Encrantz, J. C. \& Sjövall, J. (1959) Clin. Chim. Acta 4, 793-799.

7. Palmer, R. H. (1967) Proc. Nat. Acad. Sci. USA 58, $1047-1050$.
8. Iwata, T. \& Yamasaki, K. (1964) J. Biochem. 56, 424-431. 9. Nair, P. P. \& Kritchevsky (1971) The Bile Acids, Vol. 1-3, Plenum Press, New York, London.

10. Wildgrube, H. J., Müller, K. H. \& Winkler, M. (1980) HepatoGastroenterology 27, Suppl. 302.

11. Demers, L. M. \& Hepner, G. H. (1976) Am. J. Clin. Pathol. 66, 831-839.

Priv.-Doz. Dr. H. J. Wildgrube Zentrum der Inneren Medizin Theodor-Stern-Kai 7

D-6000 Frankfurt/Main 70 